\title{
Telescope Array Composition Summary
}

\section{William Hanlon ${ }^{* a}$, Daisuke lkeda ${ }^{b}$, Jon Paul Lundquist ${ }^{a}$, Thomas Stroman ${ }^{a}$ and Yana Zhezher ${ }^{c}$ for the Telescope Array Collaboration ${ }^{d}$}

${ }^{a}$ High Energy Astrophysics Institute \& Department of Physics and Astronomy, University of Utah, Salt Lake City, UT, USA

${ }^{b}$ Institute for Cosmic Ray Research, University of Tokyo, Kashiwa, Chiba, Japan

${ }^{c}$ Institute for Nuclear Research of the Russian Academy of Sciences, 60th October Anniversary St. 7a, Moscow 117312, Russia

${ }^{c}$ http://www. telescopearray.org/index.php/research/collaborators

E-mail: whanlonecosmic.utah.edu

Ultra high energy cosmic ray (UHECR) chemical composition is important to resolving questions about the locations of UHECR sources and propagation models. Because composition can only be deduced by a process of statistical inference via the observation of air shower maxima $\left(X_{\max }\right)$, UHECR observatories with large data collection rates must be employed to reduce statistical fluctuations. Telescope Array (TA), the largest cosmic ray observatory in the Northern Hemisphere, is designed to answer the question of UHECR composition, as well as other important features of cosmic ray flux, by combining a large array of over 500 scintillation surface detectors spread over $700 \mathrm{~km}^{2}$, and three fluorescence detector stations overlooking the array. With eight years of data recorded, results of the measurements of UHECR composition are presented. UHECR composition is traditionally measured by comparing the first and second moments of the distributions of shower maxima, which evolves with energy, between data and simulations. Reducing statistical fluctuations in the data helps to distinguish between different primary elements in the flux. In the current generation of cosmic ray observatories, UHECR data sets are large enough, and statistical uncertainties are now small enough, that we can safely distinguish between very light primary source flux (i.e., protons) and heavy flux (i.e., iron). Reducing systematic uncertainties is also important though, since large systematic shifts in air shower maxima will influence the interpretation of the data when compared to models. TA therefore employs different methods of measuring $X_{\max }$, including stereo air fluorescence, air fluorescence-surface counter hybrid, and a new technique using only surface counters. Updated results of TA hybrid composition among the different methods are presented using up to eight years of data. Agreement among all TA hybrid composition results are shown as well as detailed systematic errors which can be further explored by comparing composition results of the different measurement methods. Comparison of TA $X_{\max }$ data are compared to different composition models as well.

35th International Cosmic Ray Conference - ICRC2017

10-20 July, 2017

Bexco, Busan, Korea

* Speaker. 


\section{Introduction}

\subsection{UHECR Composition Physics}

The source of UHECRs still remains a mystery over a century after the first cosmic rays were observed by Hess in 1912. The flux of cosmic rays above the knee, $\gtrsim 10^{15} \mathrm{eV}$, displays features that provide hints about UHECR sources, their distances from Earth, and chemical composition. Models that attempt to describe this flux require composition as a fundamental input. Composition is therefore a key parameter to understanding the origin of UHECRs. Astrophysical models of cosmic ray propagation describe scenarios in which changes in the primary source energy cause a change in observed flux. Interaction models do not posit sharp changes in primary source energy and instead attempt to describe the changes in flux due to primary particle interactions with the interstellar medium or change in the acceleration mechanism of the sources themselves. Depending upon the model, these lead to predictions of flux that change based on energy and total charge ( $\sim E / Z$ ), or total mass $(\sim E / A)$, of the primary particle [1]. UHECR flux around the energy of the ankle hardens. Some models explain this feature using extragalactic protons. Models which predict galactic sources in this energy range though predict much heavier elements as the primary sources.

UHECRs can only efficiently be observed through indirect means for energies above $10^{14} \mathrm{eV}$, therefore methods that infer the composition by statistical methods are employed. This is best done by fluorescence detectors and examining the distribution of observed depth in the atmosphere where the electromagnetic component of the shower is largest. This depth is called $X_{\max }$ and is measured in $\mathrm{g} / \mathrm{cm}^{2}$. We can understand why $X_{\max }$ is related to composition by resorting to a model of extensive air showers originally developed by Heitler [2]. Heitler introduced a simple branching model of electromagnetic (EM) showers in which a high energy primary particle collides inelastically with a target particle. An EM shower is generated through the repeated processes of bremsstrahlung of $e^{ \pm}$and pair production of photons. In this simplified model at each interaction length, $\lambda$, two particles are added to the shower for each existing particle. The size of the shower $N$ at depth $X$ after $n$ interaction lengths have been traversed is therefore $N(X)=2^{n}$, or expressed in depth, $N(X)=2^{X / \lambda}$. The average energy for each particle is $E(X)=E_{0} / 2^{n}=E_{0} / 2^{X / \lambda}$, where $E_{0}$ is the energy of the primary particle. Particle production continues until the average particle energy decreases below the critical energy, $E_{\mathrm{c}} . E_{\mathrm{c}}$ is defined as the energy at which particle energy lost due to collisions exceeds radiative energy losses. At the critical energy, the shower size reaches its maximum and is denoted by $N_{\max }$ and the depth is $X_{\max }$. We find that $N_{\max }=E_{0} / E_{\mathrm{c}}$ and $X_{\max } \propto \ln \left(E_{0} / E_{\mathrm{c}}\right)$. The results for hadron induced showers are similar. For a proton initiated shower $X_{\max }$ is proportional to $\ln E_{0}$. For primary particles of mass $A$, the superposition principle allows us to treat the particle as $A$ independent nucleons, each with average initial energy of $E_{0} / A$. In this case using the Heitler model we find $X_{\max } \propto \ln \left(E_{0} / A\right)$. Heavier primary particles are therefore expected to reach shower maximum at shallower depths in the atmosphere than light primaries. Finally, the property of shower universality tells us that for showers initiated by a hadronic primary particle (of any mass) the electromagnetic energy spectra all evolve in the same way, parameterized by the shower age, $s$ [3]. In this way we can use the same method of observing $X_{\max }$ of the EM component of a shower to determine the mass of the primary particle, even if that particle is a 
proton or an iron nucleus. For more details about the treatment of hadronic showers as related to cosmic ray composition refer to [4] and [5].

\subsection{Telescope Array Detector}

Telescope Array (TA) is one of few detectors in the world able to shed light on the composition of UHECRs. It is located in Millard County Utah $\left(39.3^{\circ} \mathrm{N}\right.$ and $112.9^{\circ} \mathrm{W}, 1400 \mathrm{~m}$ above sea level), consisting of 507 scintillation surface counters sensitive to muons and electrons, and 48 fluorescence telescopes located in three fluorescence detector stations overlooking the counters. The spacing of the counters in the SD array is $1.2 \mathrm{~km}$ and they are placed over an area of approximately $700 \mathrm{~km}^{2}$. To measure $X_{\max }$ fluorescence detectors must be used. For those events that also have their arrival time simultaneously measured by the SD array though, the geometry of an individual shower can be very well measured. These hybrid events are very valuable for use in high quality measurements of $X_{\max }$. Telescope Array began hybrid data collection in May 2008 and currently has analyzed over eight years of data using this method. Another method to achieve excellent resolution in reconstructed shower geometry is to observe showers using multiple fluorescence detectors at different sites. This method of stereo observation is also being undertaken by TA. The results of three different measurements of $X_{\max }$ using hybrid reconstruction techniques and one using the stereo technique are presented in this paper. Information describing the details of the construction and operation of the TA surface detectors and fluorescence detectors can be found in $[6,7,8]$.

\section{TA Composition Analysis Methods}

\subsection{BR/LR Hybrid Composition}

Measurement of $X_{\max }$ requires a fluorescence detector operating in clear, dark sky conditions, so that the evolution of the size of the electromagnetic component of the shower can be observed. Utilizing a single FD eye though is not sufficient to make an accurate measurement of $X_{\max }$ for most showers. Fluorescence detectors measure the times and amount of light from a shower very well in the shower-detector plane, because the distance to the showers and the relatively small pixel size of the detector causes the shower to appear as a line source. From the tube trigger times and the well known pointing directions of the tubes, the distance to the shower $\left(R_{\mathrm{p}}\right)$ and the angle of the shower in the plane $(\psi)$ are found through fitting. The monocular reconstructed resolution of $R_{\mathrm{p}}$ and $\psi$ is often too poor to be used to well place the shower's true track in the atmosphere, and therefore the depth of $X_{\max }$ is not well measured. If one combines the arrival time and core location as measured by a second independent detector, such as an array of surface detectors, the shower geometry can then be very well measured given these additional constraints. Such a measurement of $X_{\max }$ is called a hybrid measurement.

Telescope Array has three independent hybrid measurements of $X_{\max }$ : two utilizing the Black Rock Mesa and Long Ridge FD stations, and one published result utilizing the Middle Drum FD station. Middle Drum was built using repurposed hardware from the HiRes experiment. The electronics of Middle Drum use a sample and hold technique to calculate; each event trigger provides a single ADC readout of total light accumulated by each tube. The Middle Drum FD is located about 
$8 \mathrm{~km}$ away from the border of the SD array. This distance can affect the hybrid aperture, especially for low energy events, since hybrid analysis requires coincidence between FD and SD triggering. Middle Drum uses mirrors with $5.1 \mathrm{~m}^{2}$ collection area constructed of 4 clover leaf segments.

The Black Rock Mesa and Long Ridge FD detectors were newly constructed for the TA experiment. They use FADC electronics with a sampling rate of $10 \mathrm{MHz}$, allowing us to measure the development of a shower in a single tube over time. These FD stations are closer to the SD array border ( $3 \mathrm{~km}$ for BR and $4 \mathrm{~km}$ for LR) and they use larger mirrors, $6.8 \mathrm{~m}^{2}$ collection area utilizing 18 hexagonal mirror segments, than Middle Drum. These differences between BR/LR and MD design affect the triggering and reconstruction of hybrid events for energies where the acceptance is not fully efficient. For this reason, the $X_{\max }$ analyses of Middle Drum and Black Rock/Long Ridge have been done separately. In this section the results of Black Rock and Long Ridge hybrid analysis will be presented.

Black Rock and Long Ridge hybrid analyses have been performed independently by two authors (Hanlon and Ikeda, hereinafter referred to as analysis A and analysis B, respectively), so that the algorithms, software, Monte Carlo development, and data processing are each done by one of us. This is useful in understanding systematic differences that can arise from seemingly simple choices about air shower reconstruction, such as treatment of the atmosphere, FADC signal processing, shower parameterization, and Monte Carlo generation, to name a few. BR/LR hybrid $X_{\max }$ measurements are TA's highest statistics measurements of composition, the highest number of events collected is over 3000. For energies $>10^{18.2} \mathrm{eV}$, the total $X_{\max }$ bias for QGSJet II-03 and QGSJet II-04 protons in less than $1 \mathrm{~g} / \mathrm{cm}^{2}$ and for QGSJet II-03 and QGSJet II-04 iron it is less than $4 \mathrm{~g} / \mathrm{cm}^{2} . X_{\max }$ resolution is no greater than $20 \mathrm{~g} / \mathrm{cm}^{2}$ for protons and no greater than $15 \mathrm{~g} / \mathrm{cm}^{2}$ for iron. Energy resolution for protons is less than $6 \%$ and for iron it is less than $4 \%$. Figure 1 shows a comparison BR/LR hybrid data for the two different analysis efforts and reconstructed Monte Carlo for QGSJet II-03 and QGSJet II-04 protons, helium, nitrogen, and iron in four different energy ranges.

A two-sample Kolmogorov-Smirnov test carried out on the two data distributions returns probabilities of $0.102,0.568,0.541,0.759$, indicating they are all accepted at the $95 \%$ confidence level. It's important to note that, though these analyses are conducted independently, the observed data is correlated. The data distribution of analysis A, as well as all Monte Carlo distributions shown, are normalized to analysis B data in this figure.

\subsection{Hybrid Composition}

Results of $X_{\max }$ measurements were first published in 2015 utilizing the Middle Drum fluorescence detector [9]. This was a hybrid measurement spanning five years of data collection. This analysis utilizes a reconstruction technique dubbed "pattern recognition" which attempts to identify air shower tracks that have a clear rise and fall in their profile. This is important to reduce the energy dependence of $X_{\max }$ and for a precise measurement of $X_{\max }$, since the shower maximum must be observed to accurately fit the shower profile. Also, some additional amount of shower before and after $X_{\max }$ is required to minimize uncertainties on that fit. Middle Drum analysis measures $X_{\max }$ for energies above $10^{18.4} \mathrm{eV}$ and the five year study collected 438 events. Resolution and biases are similar to those of the BR/LR hybrid $X_{\max }$ analyses: $X_{\max }$ resolution is $\sim 22 \mathrm{~g} / \mathrm{cm}^{2}$, reconstruction bias is $<2 \mathrm{~g} / \mathrm{cm}^{2}$, and energy resolution is $7 \%$. 


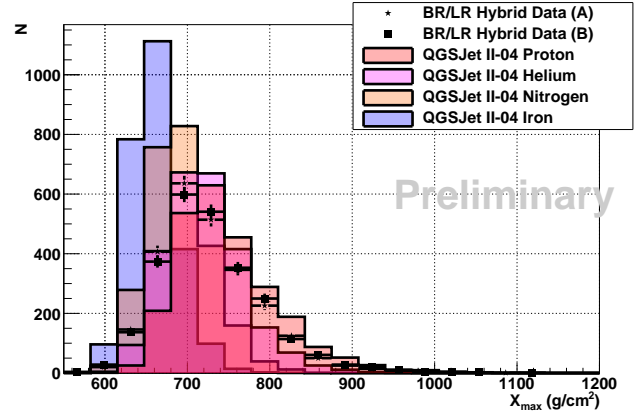

(a) $18.2 \leq \log _{10}(E / \mathrm{eV})<18.6$

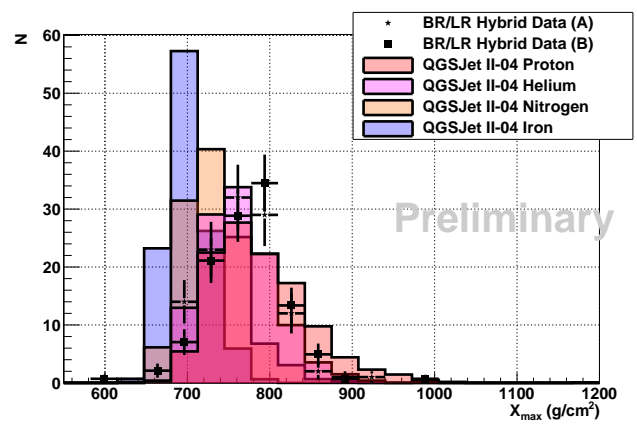

(c) $19.0 \leq \log _{10}(E / \mathrm{eV})<19.4$

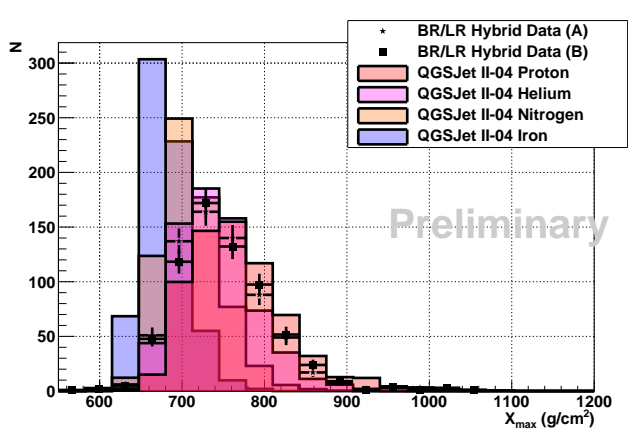

(b) $18.6 \leq \log _{10}(E / \mathrm{eV})<19.0$

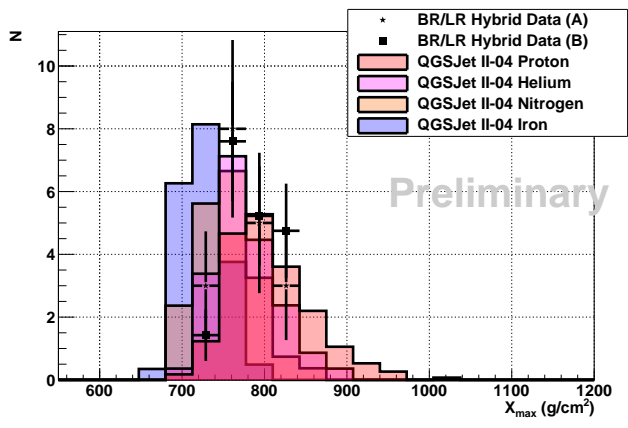

(d) $\log _{10}(E / e V) \geq 19.4$

Figure 1: Preliminary 8 year BR/LR hybrid $X_{\max }$ data of two independently performed analyses compared to several different reconstructed Monte Carlo models.

This analysis has recently been extended to seven years of data, collecting an additional 175 events [10], shown in Figure 2.

\subsection{Stereo Composition}

The stereo technique uses multiple fluorescence detectors to measure an air shower profile. TA has three FD stations, so a single shower of sufficient energy and geometry may be viewed simultaneously by up to all three detectors, though more commonly only two detectors are used. TA FD stations are all equally distant, $21 \mathrm{~km}$, from a central point of the SD array. When a shower is observed by multiple detectors at different sites, each detector constructs locally a shower-detector plane. Multiple planes crossing each other constrain the air shower track tightly, providing another reconstruction method to accurately determine the geometry. Figure 3 shows mean $X_{\max },\left\langle X_{\max }\right\rangle$, measured using the stereo technique over eight years. Stereo reconstructed $\left\langle X_{\max }\right\rangle$ of QGSJet II-04 proton and iron are also shown as well.

\subsection{SD Composition}

The techniques presented thus far use fluorescence detectors which observe $X_{\max }$ and composition has been inferred by comparing the moments and distributions of $X_{\max }$ to models. Another method of measuring composition is employed at TA using only the SD array. This method uses multivariate analysis (MVA) and a boosted decision tree (BDT) using thirteen observables sensitive 

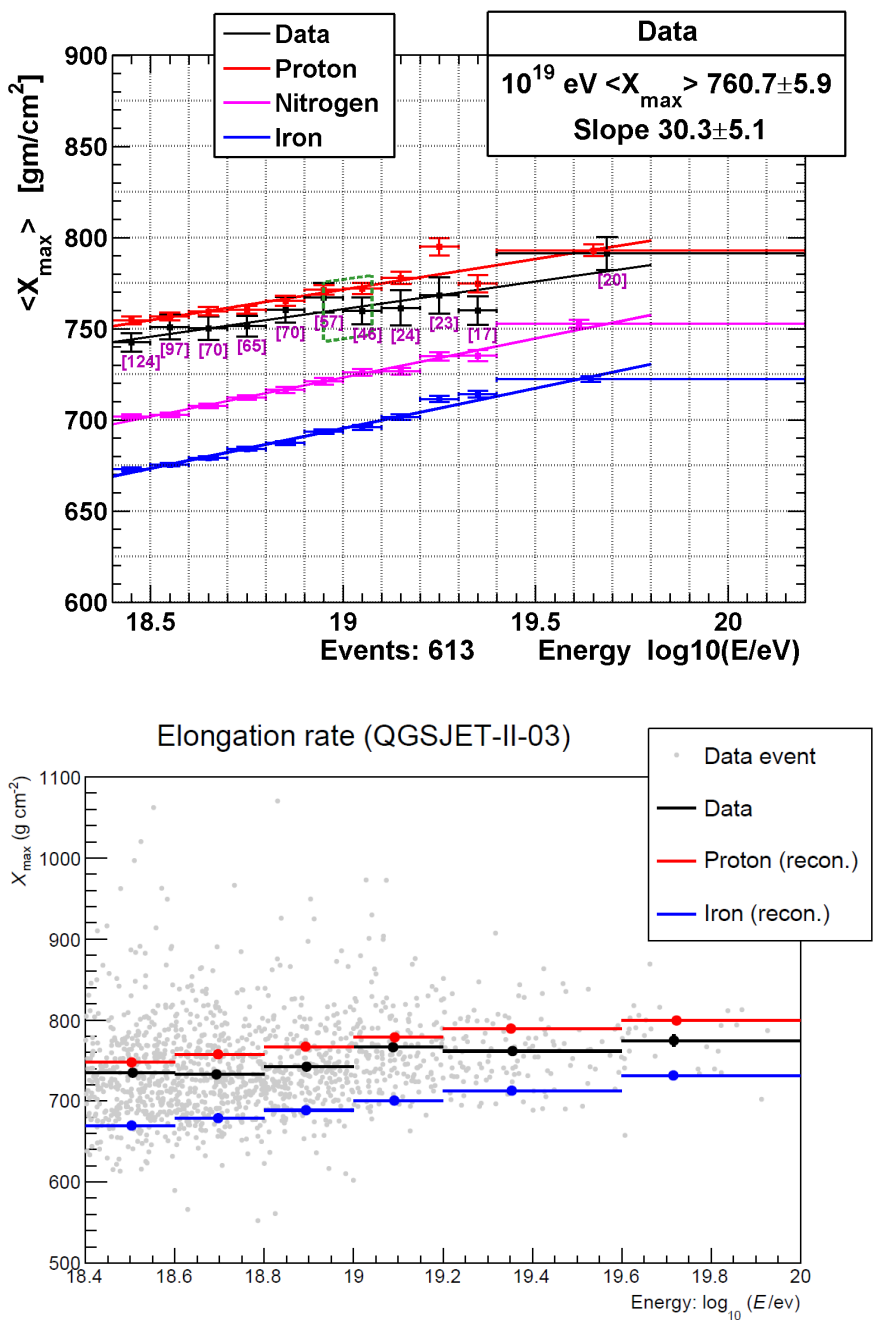

Figure 2: Seven years of Middle Drum hybrid $X_{\max }$ measurements for $\log _{10}(E)>18$.4. MD hybrid $X_{\max }$ for simulated proton, nitrogen, and iron are compared as well.

Figure 3: Preliminary eight year stereo composition measurement. Events reconstructed in stereo use multiple fluorescence detector stations observing a shower simultaneously. The expected elongation rate for simulated QGSJet II-03 proton and iron reconstructed by stereo observation is shown as well.

to composition (i.e., Linsley front curvature parameter, number of SDs hit, etc.) to discriminate cosmic ray primaries by calculating a single parameter, $\xi$, and trained using a model dependent two-component Monte Carlo (proton and iron). For each energy bin being observed, $\xi$ is calculated for data, proton, and iron MC. The proton and iron MC are mixed in varying proportions and a KS test is performed for $\xi$ between data and the mixture. The minimum value of the KS distance between data and mixture is used to determine the mixture of proton and iron that best matches data and the average atomic mass.

\subsection{TA $X_{\max }$ Comparison}

Figure 5 compares $\left\langle X_{\max }\right\rangle$ of the various measurements. The gray band indicates systematic uncertainties of $20.3 \mathrm{~g} / \mathrm{cm}^{2}$ on BR/LR hybrid reconstruction. All measurements shown have similar systematic uncertainties. The different methods agree in $\left\langle X_{\max }\right\rangle$ within systematic uncertainties. For $E>10^{18.2} \mathrm{eV}$ the $\left\langle X_{\max }\right\rangle$ of all TA $X_{\max }$ measurements are consistent with a light composition, meaning the mean mass in all energy bins is more likely to consist predominantly of light elements such as protons and helium and not likely to consist of very much iron. There is clear separation in $\left\langle X_{\max }\right\rangle$ between the data and QGSJet II-04 nitrogen as well. The shapes of the distributions are 


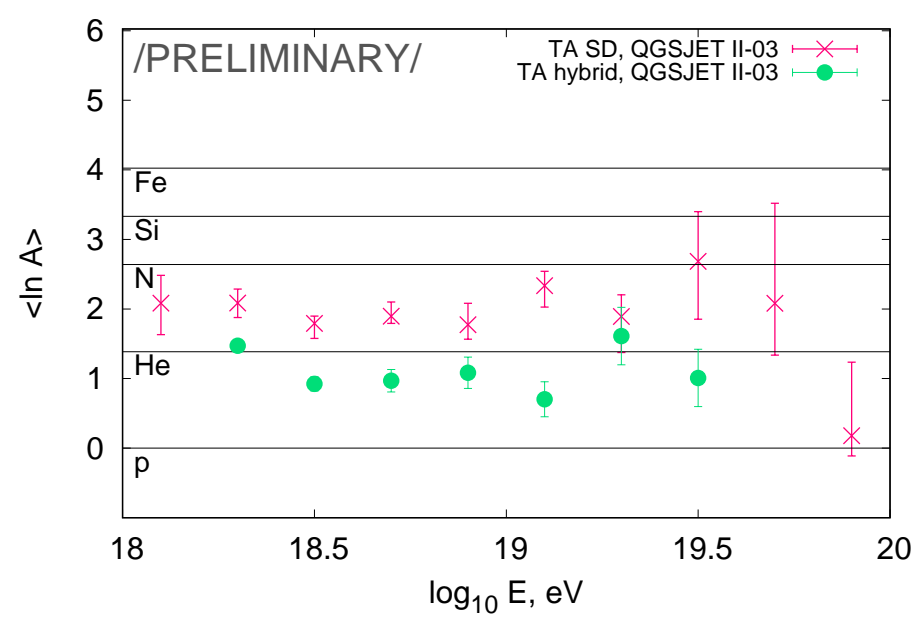

Figure 4: Mean atomic mass of data measured using only the TA SD array. This method requires a model to compare the data to. Here QGSJet II-03 is shown. The mean atomic mass of the BR/LR hybrid data is shown in comparison.

also consistent with a light composition as well (see Figure 1). For energies above $10^{19} \mathrm{eV}$ though TA's exposure is rapidly decreasing, causing a possible depletion of events in the tails of the $X_{\max }$ distributions due to statistical undersampling.

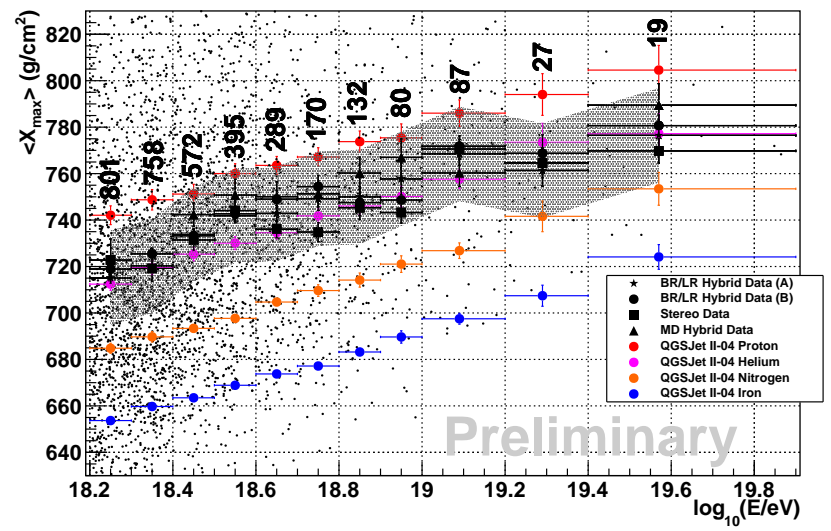

Figure 5: Comparison of four different measurements of $X_{\max }$ using TA data. The gray band are systematic uncertainties of $20.3 \mathrm{~g} / \mathrm{cm}^{2}$ on BR/LR hybrid reconstruction. Event numbers of the BR/LR hybrid data (A) all also shown.

The widths of the $X_{\max }$ distributions, $\sigma\left(X_{\max }\right)$, of the different TA analyses is shown in Figure 6. The widths are also consistent with a light composition for $10^{18.2}<E<10^{19.0} \mathrm{eV}$. The widths of the distributions are shown only up to $10^{19} \mathrm{eV}$ because TA statistics become too low above that energy to accurately measure them. If deep $X_{\max }$ tails are present in the true distributions, then high statistics are required to ensure $\sigma\left(X_{\max }\right)$ is not biased by undersampling. $\left\langle X_{\max }\right\rangle$ is less susceptible to bias by the presence a few events in tails of the distribution. In the face of very few total events in the entire distribution, even a couple of events in the tails may have a very large effect on $\sigma\left(X_{\max }\right)$. For that reason, TA does not at this time have sufficient exposure in our $X_{\max }$ analyses to have confidence in the widths of our distributions above $10^{19} \mathrm{eV}$.

\section{Conclusions}

Telescope Array employs multiple analyses combining different components of the observatory to perform high quality measurements $X_{\max }$ of UHECRs. At least seven years of data have 


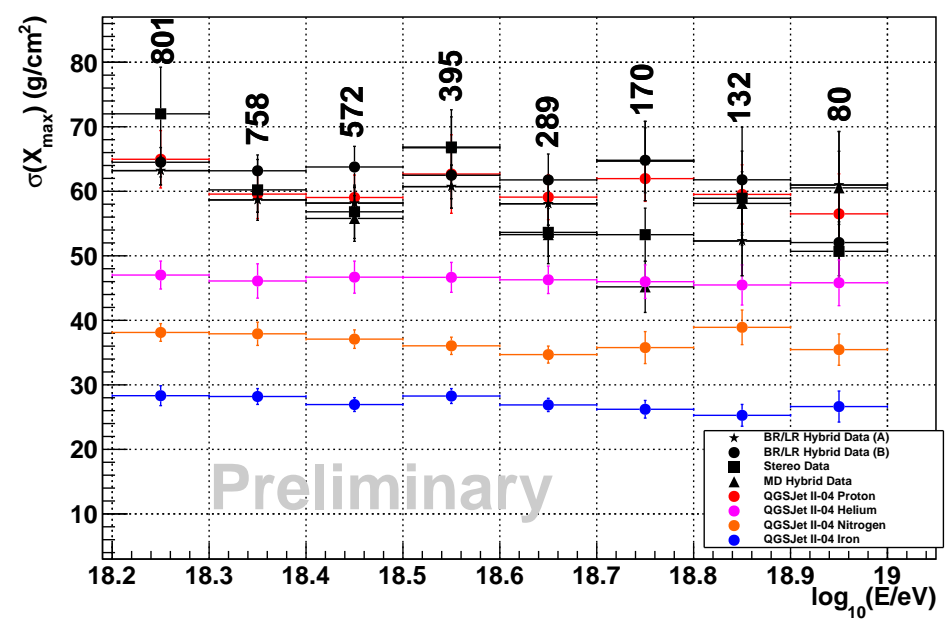

Figure 6: $\sigma\left(X_{\max }\right)$ of the different TA $X_{\max }$ analyses. Event numbers of the BR/LR hybrid (A) data are also shown.

been collected among the different methods. Agreement between the moments of $X_{\max }$ is within systematic uncertainties of the highest statistics method (BR/LR hybrid), which is also of order the $X_{\max }$ resolution of these different methods. A technique which utilizes only the SD array and MVA boosted decision tree analysis has measured the UHECR mean atomic mass. TA's observed $\left\langle X_{\max }\right\rangle$ is consistent with a light composition for $E>10^{18.2} \mathrm{eV} . \sigma\left(X_{\max }\right)$ is consistent with a light composition for $E<10^{19.0} \mathrm{eV}$. TA's statistics are limited above $10^{19} \mathrm{eV}$ preventing an interpretation of the widths beyond that energy.

\section{References}

[1] Hörandel, J. R., Models of the knee in the energy spectrum of cosmic rays, Astropart.Phys. 21, (2004) 241

[2] J.F. Carlson and J.R. Oppenheimer, On Multiplicative Showers, Phys. Rev. 51, (1937) 220

[3] Giller, M. et al., On the Cherenkov light contribution to the fluorescence of the highest energy air showers, Proceedings, 28th International Cosmic Ray Conference (ICRC 2003): Tsukuba, Japan, July 31-August 7, 2003, 2, (2003) 619

[4] Engel, R. and Heck, .D and Pierog, T., Extensive air showers and hadronic interactions at high energy, Ann. Rev. Nucl. Part. Sci. 611, (2011) 467

[5] Kampert K. and Unger, M., Measurements of the Cosmic Ray Composition with Air Shower Experiments, Astropart. Phys. 35, (2012) 660

[6] Abu-Zayyad T. et al., The surface detector array of the Telescope Array experiment, Nucl. Instrum. Meth. A689, (2013) 87

[7] Tokuno H. et al., New air fluorescence detectors employed in the Telescope Array experiment, Nucl. Intrum. Meth. A676, (2012) 54

[8] Abu-Zayyad, T., et al., Nucl. Instrum. Meth., A450, (2000) 253

[9] Abbasi, R., et al., Study of Ultra-High Energy Cosmic Ray composition using Telescope ArrayâĂŹs Middle Drum detector and surface array in hybrid mode, Astropart. Phys., 64, (2015) 49

[10] Lundquist, Jon Paul, PoS, ICRC2015, (2016) 441 